\title{
Temperature dependence of $\rho$ meson-nucleon coupling constant from the AdS/QCD soft-wall model
}

\author{
Shahin Mamedov $\odot^{1,2, *}$ and Narmin Nasibova ${ }^{2 \dagger}$ \\ ${ }^{1}$ Institute for Physical Problems, Baku State University, Z. Khalilov Street 23, Baku AZ-1148, Azerbaijan \\ ${ }^{2}$ Institute of Physics, Azerbaijan National Academy of Sciences, \\ H. Cavid Avenue 131, Baku AZ-1143, Azerbaijan
}

(Received 9 July 2021; accepted 16 July 2021; published 24 August 2021)

\begin{abstract}
We investigate the dependence of the $\rho$ meson-nucleon coupling constant on the temperature of the medium using the soft-wall model of AdS/QCD. The finite temperature profile functions for the vector and fermion fields are applied to the model having a thermal dilaton field. The interaction Lagrangian in the bulk between these fields is written as in the zero temperature case and includes minimal- and magnetictype interactions. The temperature dependence of the $g_{\rho N N}(T)$ coupling constant and its terms are plotted. We observe that the coupling constant and its separate terms become zero at the medium temperature near the Hawking temperature of the phase transition.
\end{abstract}

DOI: 10.1103/PhysRevD.104.036010

\section{INTRODUCTION}

The study of hadron quantities such as mass, decay and coupling constants, form factors, etc., at finite temperature is of great importance for the investigation of properties of hot hadronic matter obtained in the collisions of heavy ions or protons. After a correspondence was found in theory between five-dimensional gravity in anti-de Sitter spacetime and quantum field theory in four dimensions [1-4], several models and approaches were constructed in order to solve the problems in particle physics and to investigate the processes, which take place in hot hadronic matter and cannot be solved in perturbation theory. One such models is the so-called soft-wall model of the AdS/QCD correspondence [5-8]. This model has turned out to be an effective tool for the calculation of phenomenological hadron quantities [9-19], for the phase transition between hot hadronic matter and quark-gluon plasma [20], and for theoretical studies of hot hadronic matter [21-28]. The properties of mesons and baryons in the thermal medium have been investigated in the framework of different models including the hard- and soft-wall models of the AdS/QCD bottom-up approach (see, for example, [29] and references therein). The influence of the hot hadronic medium on

\footnotetext{
* Corresponding author. sh.mamedov62@gmail.com

†n.nesibli88@gmail.com

Published by the American Physical Society under the terms of the Creative Commons Attribution 4.0 International license. Further distribution of this work must maintain attribution to the author(s) and the published article's title, journal citation, and DOI. Funded by SCOAP ${ }^{3}$.
}

interactions between hadrons is one of the topical questions of elementary particle physics in the hot nuclear medium. In particular, knowledge of the temperature dependence of the coupling constants and form factors of the strong interactions between mesons and baryons surrounded by hot matter will help us to understand the interactions between particles in this medium. AdS/QCD models are useful for study of this question as well. It is known that quantum field theory at finite temperature in a confined phase is holographically dual to gravitational theory in the AdS-Schwarzschild metric. The soft-wall AdS/QCD model at finite temperature is based on this gravity background. In addition to this thermalization, in the thermal soft-wall model in $[23,25,26]$ the dilaton field, which is thermal as well, was considered. This suggestion provides two sources for conformal symmetry breaking and an explicit form of such a dilaton was established using the thermal loop approach in a field theory [25]. Solutions of the equations of motion (profile functions) for the boson and fermion fields interacting with the thermal dilaton field were given in Refs. $[25,26]$. As an application of this model, the hadron form factors and the electromagnetic properties of nucleons and Roper resonances at finite temperature were studied using this approach in Ref. [27]. We are interested in the temperature dependence of the interactions between the hadrons. More concretely, we want to know how much the screening constants of the meson-baryon couplings in hot hadronic matter will depend on temperature prior to the confinement-deconfinement phase transition. Having explicit profile functions for the hadrons and using the holographic technique, one can easily solve this problem in the framework of this thermal dilaton soft-wall model. Here we consider the simplest of these coupling constantsnamely, the $\rho$ meson-nucleon coupling constant in the 
framework of this approach-and investigate the dependence of this constant when the temperature approaches the phase transition temperature.

The remainder of this paper is organized as follows: In Sec. II we introduce the soft-wall model at finite temperature. In Secs. III and V we obtain the bulk-to-boundary propagators for the free vector, scalar, and spinor fields in the bulk at finite temperature. In Sec. IV we develop the idea of the chiral condensate and the breaking of chiral symmetry at finite temperature. In Sec. VI we write the Lagrangian for the vector-spinor interaction in the bulk and, using holographic correspondence, obtain the temperaturedependent integral expression for the $\rho$ meson-nucleon coupling constant in boundary QCD theory. In Sec. VII we fix parameters and plot graphics for the dependence of the coupling constant on the temperature, and in Sec. VIII we discuss our results.

\section{SOFT-WALL MODEL AT FINITE TEMPERATURE}

In general, in the soft-wall model of AdS/QCD at finite temperature, the dilaton field $\varphi(z)$ can be considered the temperature-dependent one, $\varphi(z, T)$, and the action for this model will be written in terms of such a dilaton:

$$
S=\int d^{4} x d z \sqrt{g} e^{-\varphi(z, T)} L(x, z, T)
$$

Here $g$ denotes $g=\left|\operatorname{det} g_{M N}\right|(M, N=0,1,2,3,5)$ and the extra dimension $z$ varies in the range $0<z<\infty$. An exponential factor was introduced to make the integral over the $z$ coordinate finite at the IR boundary $(r \rightarrow \infty)$, and the $k$ parameter is a scale parameter of a few hundred $\mathrm{MeV}$. According to the case with the AdS/CFT correspondence at finite temperature, the boundary field theory corresponds to the gravity theory given by the AdSSchwarzschild metric [2]:

$$
\begin{aligned}
d s^{2} & =e^{2 A(z)}\left[-f(z) d t^{2}-(d \vec{x})^{2}-\frac{d z^{2}}{f(z)}\right], \\
f(z) & =1-\frac{z^{4}}{z_{H}^{4}},
\end{aligned}
$$

where $z_{H}$ is the position of the event horizon and is related to the Hawking temperature as $T=1 /\left(\pi z_{H}\right), x=(t, \vec{x})$ is the set of Minkowski coordinates, $A(z)=\log \left(\frac{R}{z}\right)$, and $R$ is the AdS radius.

For convenience, in $[25,26]$ the Regge-Wheeler tortoise coordinate $r$,

$$
r=\int \frac{d z}{f(z)},
$$

was applied as a fifth one instead of $z$, and the terms of higher order than $T^{8}$ in the expansion of $z$ were neglected.
This gives the following relation between the $r$ and $z$ coordinates:

$$
r \approx z\left[1+\frac{z^{4}}{5 z_{H}^{4}}+\frac{z^{8}}{9 z_{H}^{8}}\right] .
$$

The metric for the AdS-Schwarzschild space-time in these coordinates will be written as

$$
d s^{2}=e^{2 A(r)} f^{\frac{3}{5}}(r)\left[d t^{2}-\frac{(d \vec{x})^{2}}{f(r)}-d r^{2}\right],
$$

with $A(r)=\log \left(\frac{R}{r}\right)$. The thermal factor $f(r)$ in terms of the $r$ coordinate has the same form as in Eq. (2):

$$
f(r)=1-\frac{r^{4}}{r_{H}^{4}} .
$$

In the approach for the finite temperature soft-wall model in Refs. [25-27] the dilaton field $\varphi=k^{2} z^{2}$ is considered a temperature-dependent one when the temperaturedependent dilaton parameter $k^{2}$ is introduced:

$$
\varphi(r, T)=K^{2}(T) r^{2} .
$$

This thermal form of the dilaton parameter has been established by means of the relation between $k^{2}$ and the quark condensate $\Sigma$. It was proposed that the relation between these constants holds in a finite temperature case, and thus the temperature dependence of the condensate $\Sigma(T)$, which is known from chiral perturbation theory, determines the temperature dependence of the dilaton parameter $K^{2}(T)$. In such a way $K^{2}(T)$ was finally found in the following form [25]:

$$
K^{2}(T)=k^{2}[1+\rho(T)] .
$$

So, $K^{2}(T)$ is the parameter of spontaneous breaking of chiral symmetry and the temperature-dependent term $\rho(T)$ up to $T^{4}$ order was established in the form [25]

$$
\rho(T)=\delta_{T_{1}} \frac{T^{2}}{12 F^{2}}+\delta_{T_{2}}\left(\frac{T^{2}}{12 F^{2}}\right)^{2} .
$$

Here $F$ is the pseudoscalar decay constant in the chiral limit and the coefficients $\delta_{T_{1}}$ and $\delta_{T_{2}}$ are defined by the number of quark flavors $N_{f}$ as follows:

$$
\delta_{T_{1}}=-\frac{N_{f}^{2}-1}{N_{f}}
$$

and

$$
\delta_{T_{2}}=-\frac{N_{f}^{2}-1}{2 N_{f}^{2}} .
$$




\section{THE MESON PROFILE FUNCTION AT FINITE TEMPERATURE}

Let us briefly present here the derivation of the profile function for mesons in the model with the thermal dilaton, which was described in [25] in detail. The vector field $M_{N}(x, r, T)$, which on the ultraviolet boundary of spacetime $(r=0)$ gives the wave function of the vector meson, is composed from the gauge fields $A_{L}$ and $A_{R}: M_{N}=$ $1 / 2\left(A_{L}+A_{R}\right)$. These gauge fields transform under the flavor symmetry subgroups $S U(2)_{L}$ and $S U(2)_{R}$, respectively, which are part of the $S U(2)_{L} \times S U(2)_{R}$ flavor group of the model. From these chiral gauge fields can be composed an axial vector field as well, which we do not consider here. The action for the scalar and vector fields in the AdS-Schwarzschild space-time in the general reads as

$$
\begin{aligned}
S_{M}= & -\frac{1}{2} \int d^{4} x d r \sqrt{g} e^{-\varphi(r, T)}\left[\partial_{N} M_{N}(x, r, T) \partial^{N} M^{N}(x, r, T)\right. \\
& \left.-\left(\mu^{2}(r, T)+V(r, T)\right) M_{N}(x, r, T) M^{N}(x, r, T)\right] .
\end{aligned}
$$

Here $V(r, T)$ is the thermal dilaton potential, and it is expressed as

$$
V(r, T)=\frac{e^{-2 A(r)}}{f^{\frac{3}{5}}(r)}\left[\varphi^{\prime \prime}(r, T)+\varphi^{\prime}(r, T) A^{\prime}(r)\right],
$$

where the prime denotes the $r$ derivative. The temperaturedependent bulk "mass" $\mu(r, T)$ of the boson field $M_{N}$ is related to that at zero temperature as follows:

$$
\mu^{2}(r, T)=\frac{\mu^{2}}{f^{\frac{3}{5}}(r)} .
$$

The five-dimensional mass $\mu^{2}$ is expressed by means of the conformal dimension $\Delta=N+L$ of the interpolating operator dual to the meson. $N$ is the number of partons and $L=\max \left|L_{z}\right|$ is the quark orbital angular momentum. $N=$ 2 for our $\rho$ meson and $L=0$ for the meson ground state. For this meson the spin $J$ is $J=1$ and the expression for $\mu^{2} R^{2}$ obtains the simple form [25]

$$
\mu^{2} R^{2}=(\Delta-1)(\Delta-3) .
$$

The axial gauge $M_{z}(x, r, T)=0$ is chosen for the vector field $M_{N}$, and the Kaluza-Klein (KK) expansion is performed as follows:

$$
M_{\mu}(x, r, T)=\sum_{n} M_{\mu n}(x) \Phi_{n}(r, T) .
$$

Here $M_{\mu n}(x)$ are KK modes wave functions corresponding to meson states, $\Phi_{n}(r, T)$ are their temperature-dependent profile functions, and $n$ is the radial quantum number.
Equation of motion for the vector field will be reduced to the Schrödinger-type equation with the following replacement: $\phi_{n}(r, T)=e^{-\frac{B_{T}(r)}{2}} \Phi_{n}(r, T)$, with $B_{T}(r)=$ $\varphi(r, T)-A(r)$. In the rest frame of the vector field the equation of motion (e.o.m.) will give us the following equation for the $\phi_{n}(r, T)$ profiles:

$$
\left[-\frac{d^{2}}{d r^{2}}+U(r, T)\right] \phi_{n}(r, T)=M_{n}^{2}(T) \phi_{n}(r, T) .
$$

Here $U(r, T)$ is the effective potential and is the sum of the temperature-dependent and nondependent parts:

$$
U(r, T)=U(r)+\Delta U(r, T) .
$$

Explicit forms of the $U(r)$ and $\Delta U(r, T)$ terms were given as

$$
\begin{gathered}
U(r)=k^{4} r^{2}+\frac{\left(4 m^{2}-1\right)}{4 r^{2}}, \\
\Delta U(r, T)=2 \rho(T) k^{4} r^{2} .
\end{gathered}
$$

Here $m=N+L-2$ and, for the $\rho$ meson with two partons, it equals $m=L$. In the low temperature case the meson mass spectrum $M_{n}^{2}$ is written as the following sum of zero and finite temperature parts:

$$
\begin{gathered}
M_{n}^{2}(T)=M_{n}^{2}(0)+\Delta M_{n}^{2}(T), \\
\Delta M_{n}^{2}(T)=\rho(T) M_{n}^{2}(0)+\frac{R \pi^{4} T^{4}}{k^{2}}, \\
M_{n}^{2}(0)=4 k^{2}\left(n+\frac{m+1}{2}\right), \quad R=(6 n-1)(m+1) .
\end{gathered}
$$

Finally, the solution of Eq. (16) for the bulk profile $\phi_{n}(r, T)$ was given in the following form [25]:

$\phi_{n}(r, T)=\sqrt{\frac{2 \Gamma(n+1)}{\Gamma(n+m+1)}} K^{m+1} r^{m+\frac{1}{2}} e^{-\frac{K^{2} r^{2}}{2}} L_{n}^{m}\left(K^{2} r^{2}\right)$.

This solution coincides with the one in the zero temperature case [8] with the replacements $z \rightarrow r$ and $K(T) \rightarrow k$ in it.

\section{BREAKING OF CHIRAL SYMMETRY AT FINITE TEMPERATURE}

The pseudoscalar field $X$, which transforms under the bifundamental representation of $S U(2)_{L} \times S U(2)_{R}$, is introduced into the AdS/QCD models in order to perform breaking of this chiral symmetry group of the model using 
the Higgs mechanism in [30-33]. The action for this field has the form

$$
S_{X}=\int d^{4} x d r \sqrt{g} e^{-\varphi(r, T)} \operatorname{Tr}\left[|D X|^{2}+3|X|^{2}\right] .
$$

Here $D^{M}$ is the covariant derivative, which includes the minimal couplings with the $A_{L, R}^{M}$ gauge fields,

$$
\begin{aligned}
D^{M} X & =\partial^{M} X-i A_{L}^{M} X+X A_{R}^{M} \\
& =\partial^{M} X-i\left[M_{M}, X\right]-i\left\{A_{M}, X\right\} .
\end{aligned}
$$

Since here we deal only with the vector field, we ignore the last term in Eq. (25). The solution of the equation of motion for the $X$ field at zero temperature was widely described in earlier works and we shall not repeat it here. We shall just recall the following vacuum expectation value for this field, which was found in the Ref. [30]:

$$
\langle X\rangle=\frac{1}{2} a m_{q} z+\frac{1}{2 a} \Sigma z^{3}=v(z) .
$$

Here $m_{q}$ is the mass of the $u$ and $d$ quarks, $\Sigma=\langle 0|\bar{q} q| 0\rangle$ is the value of the chiral condensate at zero temperature, and the constant $a=\sqrt{N_{c}} /(2 \pi)$. When the thermal case of this solution was considered in Ref. [25], the temperature was taken into account by replacing the cold condensate with the thermal one and the $z$ coordinate with $r$ :

$$
\langle X(r, T)\rangle=\frac{1}{2} a m_{q} r+\frac{1}{2 a} \Sigma(T) r^{3}=v(r, T) .
$$

In [25-27] it was supposed that the temperature dependence of the $\Sigma(T)=\langle 0|\bar{q} q| 0\rangle_{T}$ quark condensate is identical to the temperature dependence of the dilaton parameter $K^{2}(T)$ :

$$
K^{2}(T)=k^{2} \frac{\Sigma(T)}{\Sigma} .
$$

In addition, it was conjectured that the relation at zero temperature between the quark condensate $\Sigma$ and the number of flavors $N_{f}$, the condensate parameter $B$, and the pseudoscalar meson decay constant $F$ in the chiral limit

$$
\Sigma=-N_{f} B F^{2}
$$

holds for the finite temperature case as well:

$$
\Sigma(T)=-N_{f} B(T) F^{2}(T) .
$$

Then according to Eqs. (7) and (27) we can write [25]

$$
\Sigma(T)=\Sigma[1+\rho(T)] .
$$

Let us note that the relation (29) is valid until $T^{6}$ degrees of temperature. The $F(T)$ and $B(T)$ dependencies were studied in [25].

\section{NUCLEON PROFILE FUNCTION AT FINITE TEMPERATURE}

In the AdS/QCD models we have to introduce two bulk fermion fields $\left(N_{1}, N_{2}\right)$ in order to describe two independent chiral components of nucleons [31,32] on the boundary. Let us present the solution of the equation of motion for the fermion fields describing boundary nucleons in this thermal soft-wall model. The action for the thermal fermion field $N(x, r, T)$ is written as [26]

$S=\int d^{4} x d r e^{-\varphi(r, T)} \sqrt{g} \bar{N}(x, r, T) D_{ \pm}(r) N(x, r, T)$,

where $D_{ \pm}(r)$ denotes the covariant derivative and has the explicit form

$$
\begin{aligned}
D_{ \pm}(r)= & \frac{i}{2} \Gamma^{M}\left[\partial_{M}-\frac{1}{4} \omega_{M}^{a b}\left[\Gamma_{a} \Gamma_{b}\right]\right] \\
& \mp\left[\mu_{F}(r, T)+U_{F}(r, T)\right] .
\end{aligned}
$$

Here $\mu_{F}(r, T)$ is the five-dimensional mass of the thermal fermion field $N(x, r, T)$, and it is expressed in terms of $f(r, T)$ as follows:

$$
\mu_{F}(r, T)=\mu_{F} f^{\frac{3}{10}}(r, T) .
$$

Zero temperature mass $\mu_{F}$ is determined by the following equation between the number of partons $\left(N_{B}=3\right)$ in the composite fermion and the orbital angular momentum $L$ ( $L=0$ for the nucleons considered here):

$$
\mu_{F}=N_{B}+L-\frac{3}{2} .
$$

The temperature-dependent potential $U_{F}(r, T)$ in Eq. (31) for the fermions is related to the zero temperature one as follows:

$$
U_{F}(r, T)=\varphi(r, T) / f^{\frac{3}{10}}(r, T),
$$

and the nonzero components of the spin connection $\omega_{M}^{a b}$ are given by

$$
\omega_{M}^{a b}=\left(\delta_{M}^{a} \delta_{r}^{b}-\delta_{M}^{b} \delta_{r}^{a}\right) r f^{\frac{1}{5}}(r, T) .
$$

The Dirac matrices $\Gamma^{A}$ in the $\left[\Gamma^{M}, \Gamma^{N}\right]$ commutator in Eq. (31) are related to those in the reference frame by the $\Gamma^{M}=e_{a}^{M} \Gamma^{a}$ relation, where $e_{a}^{M}=r \times \operatorname{diag}\left\{\frac{1}{f(r)}, 1,1,1\right.$, $-f(r)\}$ are the inverse vielbeins. The reference frame $\Gamma^{a}$ matrices are chosen as $\Gamma^{a}=\left(\gamma^{\mu},-i \gamma^{5}\right)$. Using the axial 
gauge $N_{5}(x, r, T)=0$ we decompose the AdS fermion field into the following left- and right-chirality components:

$$
N(x, r, T)=N^{R}(x, r, T)+N^{L}(x, r, T),
$$

which are defined as the usual ones $N^{R}(x, r, T)=\frac{1-\gamma^{5}}{2} N$, $N^{L}(x, r, T)=\frac{1+\gamma^{5}}{2} N$ with the properties $\gamma^{5} N^{L}=-N^{L}$, $\gamma^{5} N^{R}=N^{R}$. The Kaluza-Klein expansion for the $N^{L, R}$ chiral components will be written in terms of the temperature-dependent profile functions $\Phi_{n}^{L, R}(r, T)$ as follows:

$$
N^{L, R}(x, r, T)=\sum_{n} N_{n}^{L, R}(x) \Phi_{n}^{L, R}(r, T) .
$$

For the nucleons the spin is $J=\frac{1}{2}$. For this case the $\Phi_{n}^{L, R}(r, T)$ profiles will be written with the prefactors

$$
\Phi_{n}^{L, R}(r, T)=e^{-\frac{3}{2} A(r)} F_{n}^{L, R}(r, T)
$$

After the substitution of these profile functions into the equations of motion in the rest frame of nucleon $(\vec{p}=0)$, we obtain the following form of the e.o.m. [26] for the $F_{n}^{L, R}(r, T)$ profile functions:

$$
\left[\partial_{r}^{2}+U_{L, R}(r, T)\right] F_{n}^{L, R}(r, T)=M_{n}^{2}(T) F_{n}^{L, R}(r, T) .
$$

The temperature-dependent spectrum $M_{n}^{2}(T)$ has a quantization similar to that in the zero temperature case,

$$
\begin{aligned}
M_{n}^{2}(T) & =4 K^{2}(T)\left(n+m+\frac{1}{2}\right) \\
& =4 k^{2}(1+\rho(T))\left(n+m+\frac{1}{2}\right) .
\end{aligned}
$$

$U(r, T)$ in Eq. (39) is the effective potential at finite temperature for the fermion field, and it can be decomposed into zero and finite temperature-dependent terms as follows:

$$
\begin{gathered}
U_{L, R}(r, T)=U_{L, R}(r)+\Delta U_{L, R}(r, T), \\
\Delta U_{L, R}(r, T)=2 \rho(T) k^{2}\left(k^{2} r^{2}+m \mp \frac{1}{2}\right) .
\end{gathered}
$$

Here

$$
m=N+L-\frac{3}{2} .
$$

Solutions to Eq. (39) are the following finite temperature profile functions for the nucleons [26]:

$$
\begin{aligned}
& F_{n}^{L}(r, T)=\sqrt{\frac{2 \Gamma(n+1)}{\Gamma\left(n+m_{L}+1\right)}} K^{m_{L}+1} r^{m_{L}+\frac{1}{2}} e^{-\frac{K^{2} r^{2}}{2}} L_{n}^{m_{L}}\left(K^{2} r^{2}\right), \\
& F_{n}^{R}(r, T)=\sqrt{\frac{2 \Gamma(n+1)}{\Gamma\left(n+m_{R}+1\right)}} K^{m_{R}+1} r^{m_{R}+\frac{1}{2}} e^{-\frac{K^{2} r^{2}}{2}} L_{n}^{m_{R}}\left(K^{2} r^{2}\right),
\end{aligned}
$$

where $m_{L, R}=m \pm \frac{1}{2}$. The profile functions $\Phi_{n}(r, T)$ and $F_{n}(r, T)$ obey the normalization conditions

$$
\begin{aligned}
& \left.\int_{0}^{\infty} d r e^{-\frac{3}{2} A(r)} \Phi_{m}^{L, R}(r, T) \Phi_{n}^{L, R}(r, T)\right) \\
& \quad=\int_{0}^{\infty} d r F_{m}^{L, R}(r, T) F_{n}^{L, R}(r, T)=\delta_{m n}
\end{aligned}
$$

and coincide with those for the zero temperature case with the replacements $r \rightarrow z$ and $K(r, T) \rightarrow k$.

\section{BULK INTERACTION AND FINITE TEMPERATURE COUPLING CONSTANT}

To derive the $\rho$ meson-nucleon thermal coupling constant in the AdS/CFT framework, we shall follow the calculation procedure used in the zero temperature case in Refs. [8,15,31,32]. To this end we should construct a Lagrangian for the interaction in the bulk between the thermal vector and fermion bulk fields. Then, identifying the bulk partition function in the AdS-Schwarzschild background with the one in thermal QCD, we shall obtain the expression for the thermal nucleon current interacting with the thermal vector meson. For the finite temperature soft-wall model considered here the interaction action will be an integral of $L_{\text {int }}$ multiplied by the exponent of the thermal dilaton field. The five-dimensional interaction action in the bulk of AdS-Schwarzschild space-time will be written as follows:

$$
S_{\mathrm{int}}=\int d^{4} x d r \sqrt{g} e^{-\varphi(r, T)} L_{\mathrm{int}}
$$

According to the holographic principle, the generating functional $Z_{\mathrm{AdS}}$ of the bulk theory is identical to the generating functional $Z_{\mathrm{QCD}}$ of the QCD theory on the UV boundary of this space-time:

$$
Z_{\mathrm{AdS}}=e^{i S_{\mathrm{int}}}=Z_{\mathrm{QCD}}
$$

So, to find the nucleon current (more precisely, a vacuum expectation value of the current), which interacts with the $\rho$ meson in boundary QCD theory, we can take the variation of the bulk functional $Z_{\text {AdS }}$ from the boundary value of the bulk vector field $M_{\mu}^{a}(q)$ as follows: 


$$
\left\langle J_{\mu}^{a}\right\rangle=-\left.i \frac{\delta Z_{\mathrm{AdS}}}{\delta M_{\mu}^{a}(q)}\right|_{M_{\mu}^{a}=0} .
$$

There are several kinds of interactions between the spinor and vector bulk fields, and $L_{\text {int }}$ consists of terms describing these interactions. Since we have the gauge fields in the bulk, the first term is a minimal gauge interaction term of the vector field with the current of fermions in the bulk,

$$
L_{M N N}^{(0)}(T)=\bar{N}_{1} e_{A}^{M} \Gamma^{A} M_{M} N_{1}+\bar{N}_{2} e_{A}^{M} \Gamma^{A} M_{M} N_{2} .
$$

Next, terms are connected with the bulk spinor field's five-dimensional "magnetic moments," which are described by $\Gamma^{M N}$. Four-dimensional components of this tensor correspond to the magnetic moments of fermions in the reference frame. By means of these "moments" the terms of the Lagrangian can be constructed in the bulk of space-time, which have "Lorentz," gauge, and parity invariant interactions with the vector field. The first such term is the following five-dimensional generalization of the usual four-dimensional magnetic interaction:

$$
\begin{aligned}
L_{M N N}^{(1)}(T) & =i k_{1} e_{A}^{M} e_{B}^{N}\left[\bar{N}_{1} \Gamma^{A B}\left(F_{L}\right)_{M N} N_{1}-\bar{N}_{2} \Gamma^{A B}\left(F_{R}\right)_{M N} N_{1}+\text { H.c. }\right] \\
& =i k_{1} e_{A}^{M} e_{B}^{N}\left[\bar{N}_{1} \Gamma^{A B} F_{M N} N_{1}-\bar{N}_{2} \Gamma^{A B} F_{M N} N_{1}+\text { H.c. }\right]+\text { axial vector term, }
\end{aligned}
$$

where $F_{M N}=\partial_{M} M_{N}-\partial_{N} M_{M}$ is the field strength tensor of the $M_{N}$ vector field.

The second such magnetic moment term was constructed in [32] and has the form

$$
\begin{aligned}
L_{M N N}^{(2)}(T) & =\frac{i}{2} k_{2} e_{A}^{M} e_{B}^{N}\left[\bar{N}_{1} X \Gamma^{A B}\left(F_{R}\right)_{M N} N_{2}+\bar{N}_{2} X^{+} \Gamma^{A B}\left(F_{L}\right)_{M N} N_{1}-\text { H.c. }\right] \\
& =\frac{i}{2} k_{2} e_{A}^{M} e_{B}^{N}\left[\bar{N}_{1} X \Gamma^{A B} F_{M N} N_{2}+\bar{N}_{2} X^{+} \Gamma^{A B} F_{M N} N_{1}+\text { axial vector term }\right] .
\end{aligned}
$$

In addition to the magnetic moment interaction this term includes an interaction with the $X$ field, and as a result of this interaction it changes the chirality of the fermion fields. As mentioned, the bulk scalar field $X$ was introduced as one, which changes the chirality of the boundary nucleons and is expressed with the quark condensate $\Sigma$ in the boundary theory. Interpretation of this term is the interaction between the bulk fermions and the gauge fields by means of the magnetic moments of the fermions and an interaction with the $X$ field (the background field of the condensate). As a result of such a tripartite interaction, the chirality of the fermions is changed. In boundary QCD theory this term describes the nucleon- $\rho$ meson-quark condensate coupling with the change in chirality of the nucleons. The $k_{1}$ and $k_{2}$ constants were determined in the hard-wall model in the zero temperature case [32]. Thus, the total "magnetic"-type Lagrangian is the following sum of these two terms:

$$
L_{M N N}^{\prime}(T)=L_{M N N}^{(1)}(T)+L_{M N N}^{(2)}(T) .
$$

Higher order bulk fields can be included in the Lagrangian terms; however, we neglect them in the approximation here.

Having explicit expressions of thermal profile functions of the bulk fields we can calculate the terms of thermal action in the momentum space and then take the variational derivative (47) from these terms. This variation gives us the following contribution of each Lagrangian term to the nucleon current:

$$
\left\langle J_{\mu}\left(p^{\prime}, p ; T\right)\right\rangle=g_{\rho N N}(T) \bar{u}\left(p^{\prime}\right) \gamma_{\mu} u(p),
$$

where $g_{\rho N N}(T)$ is the integral over the holographic coordinate $r$ and it corresponds to the thermal minimal coupling constant according to holographic identification of the bulk and boundary currents. Now we can write the contribution of each Lagrangian term to the $g_{\rho N N}(T)$ constant.

The contribution coming from the $L_{M N N}^{(0)}$ Lagrangian is denoted by $g_{\rho N N}^{(0) n m}(T)$ and its integral expression is equal to the following one:

$$
\begin{aligned}
g_{\rho N N}^{(0) n m}(T)= & \int_{0}^{\infty} \frac{d r}{r^{4}} e^{-K^{2} r^{2}} M_{0}(r, T)\left[F_{1 L}^{*(n)}(r, T) F_{1 L}^{(m)}(r, T)\right. \\
& \left.+F_{2 L}^{*(n)}(r, T) F_{2 L}^{(m)}(r, T)\right] .
\end{aligned}
$$

Here we have used the relations between the profile functions of the bulk fermion fields $F_{1 L}^{(s)}=F_{2 R}^{(s)}$, $F_{1 R}^{(s)}=-F_{2 L}^{(s)}$, which are correct for parity even states of the nucleons. $M_{0}(r, T)$ is the profile function of a vector meson in the ground state.

In the Lagrangian expressions (49) and (50) the $\Gamma^{M N} F_{M N}$ matrix is the sum of two kinds of terms-namely, $\Gamma^{5 \nu} F_{5 \nu}$ and $\Gamma^{\mu \nu} F_{\mu \nu}$. Since these terms have different physical meanings, it is useful to present the contributions of these terms separately. In the total Lagrangian $L_{M N N}^{\prime}(T)$ the $\Gamma^{5 \nu} F_{5 \nu}$ terms contribute to the $g_{\rho N N}$ constant, and the contribution of this term is expressed as follows: 


$$
\begin{aligned}
g_{\rho N N}^{(1) n m}(T)= & -2 \int_{0}^{\infty} \frac{d r}{r^{3}} e^{-K^{2} r^{2}} M_{0}^{\prime}(r, T)\left[k _ { 1 } \left(F_{1 L}^{*(n)}(r, T) F_{1 L}^{(m)}(r, T)\right.\right. \\
& \left.\left.-F_{2 L}^{*(n)}(r, T) F_{2 L}^{(m)}(r, T)\right)+k_{2} v(r, T)\left(F_{1 L}^{*(n)}(r, T) F_{2 L}^{(m)}(r, T)-F_{2 L}^{*(n)}(r, T) F_{1 L}^{(m)}(r, T)\right)\right] .
\end{aligned}
$$

Here the prime on $M_{n}$ denotes the derivative over $r$. The $\Gamma^{\mu \nu} F_{\mu \nu}$ term makes the following contribution to the expression:

$$
\begin{aligned}
f_{\rho N N}^{n m}(T)= & -4 m_{N} \int_{0}^{\infty} \frac{d r}{r^{3}} e^{-K^{2} r^{2}} M_{0}(r, T)\left[k_{1}\left(F_{1 L}^{*(n)}(r, T) F_{1 R}^{(m)}(r, T)-F_{2 L}^{*(n)}(r, T) F_{2 R}^{(m)}(r, T)\right)\right. \\
& \left.+k_{2} v(r, T)\left(F_{1 L}^{*(n)}(r, T) F_{2 R}^{(m)}(r, T)-F_{2 L}^{*(n)}(r, T) F_{1 R}^{(m)}(r, T)\right)\right]
\end{aligned}
$$

where $m_{N}$ is the mass of the nucleon. $f_{\rho N N}^{n m}(T)$ is interpreted as the contribution of the nucleon- $\rho$ meson interaction by means of the magnetic moment of the nucleon. The total coupling constant $g_{\rho N N}^{s . w}(T)$ is the following sum of the previous coupling constants:

$$
g_{\rho N N}^{s . w}(T)=g_{\rho N N}^{(0) n m}(T)+g_{\rho N N}^{(1) n m}(T) .
$$

The $g_{\rho N N}^{(0) n m}(T)$ coupling constant is interpreted as the "strong charge" of this interaction.

\section{NUMERICAL ANALYSIS}

The numerical analysis of the $g_{\rho N N}^{(0) n m}(T)$ coupling constant consists of a numerical calculation of the integrals for the constants $g_{\rho N N}^{(0) n m}(T), g_{\rho N N}^{(1) n m}(T)$, and $f_{\rho N N}^{n m}(T)$ and of numerically drawing their temperature dependencies by means of the Mathematica package. We present our numerical results for the choice of parameters for the two flavor $N_{f}=2$ case with the pseudoscalar decay constant in the chiral limit $F=0.87 \mathrm{GeV}$; for the three flavor $N_{f}=3$ case with $F=0.100 \mathrm{GeV}$; for the four flavor $N_{f}=4$ case with $F=0.130 \mathrm{GeV}$; and for the five flavor $N_{f}=5$ case $F=0.140 \mathrm{GeV}$. These sets of parameters were taken from [25]. We use the free parameters $k, k_{1}, k_{2}, m_{q}$, and

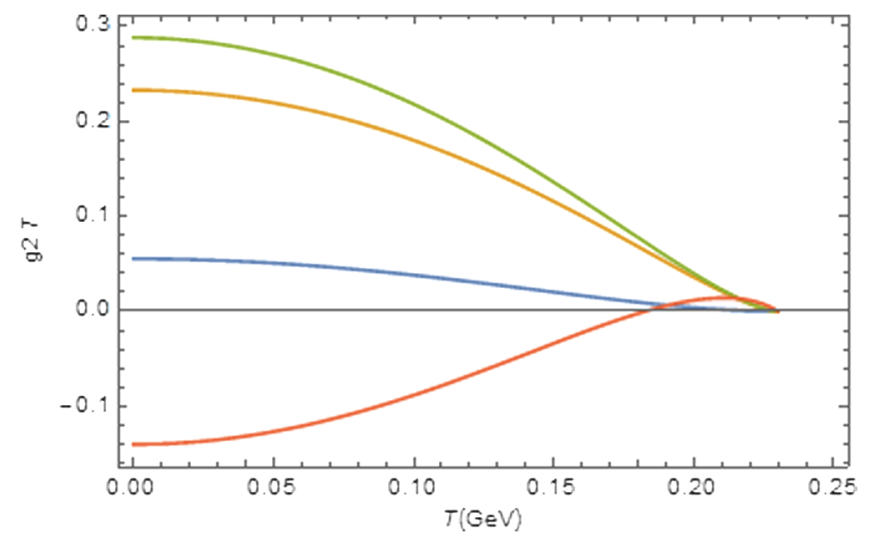

FIG. 1. Comparison of $g_{\rho N N}^{(0) n m}, g_{\rho N N}^{(1) n m}(T)$, and $f_{\rho}^{n m}(T)$ coupling constants at finite temperature for $N_{f}=2$ and $F=0.87 \mathrm{GeV}$.
$\Sigma$. The $k$ parameter was fixed at the value $k=0.383 \mathrm{GeV}$ in [25]. The parameters $k_{1}$ and $k_{2}$ were fixed at the values $k_{1}=-0.78 \mathrm{GeV}^{3}, k_{2}=0.5 \mathrm{GeV}^{3}$ in [32]. Here we do not consider these constants to be temperaturedependent ones, and we use these values in our numerical analysis. The $\Sigma=(0.368)^{3} \mathrm{GeV}^{3}$ and $m_{q}=$ $0.00145 \mathrm{GeV}$ values of these parameters were found using the fitting of the $\pi$ meson mass [34]. To have an idea of relative contributions of different terms of the Lagrangian, we present results for the temperature dependencies of the $g_{\rho N N}^{(0) n m}, g_{\rho N N}^{(1) n m}(T)$, and $f_{\rho}^{n m}(T)$ coupling constants separately. In the figures below, the blue graph curve represents the $g_{\rho N N}^{(0) n m}$ coupling constant, the orange curve shows the $g_{\rho N N}^{(1) n m}(T)$, the green curve shows the $g_{\rho N N}^{\text {(s.w.) }}(T)$, and the red one shows the $f_{\rho}^{n m}(T)$ at finite temperature. Finally, we have considered these dependencies for the first excited state $N(1440)$ of the nucleons as well. We repeat plotting graphs for the different numbers of flavors $N_{f}$ and $F$. We observe that all graphs in the Figs. 1-8 converge at one temperature value, which varies slightly in the different cases. Changing the parameter values does not change the picture in the figure and leads to only a slight deformation of the shape in the graphs.

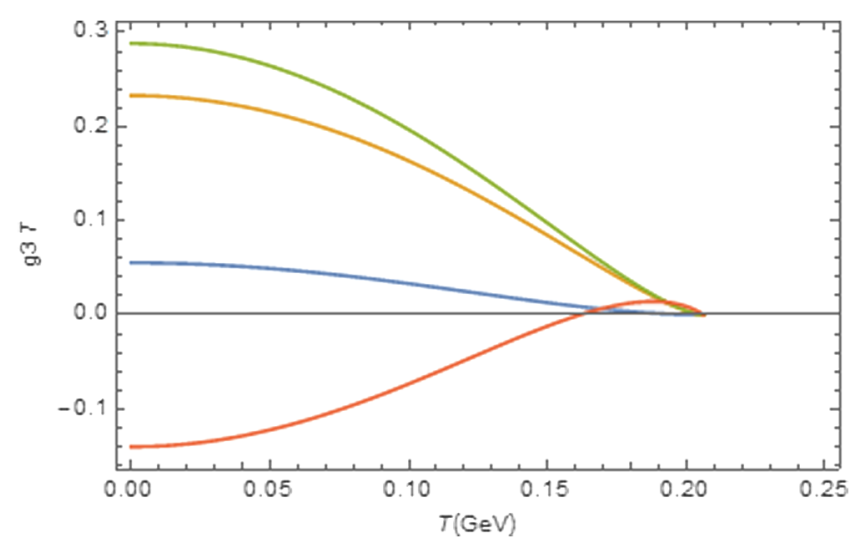

FIG. 2. Comparison of $g_{\rho N N}^{(0) n m}, g_{\rho N N}^{(1) n m}(T), g_{\rho N N}^{(\text {s.w. })}(T)$, and $f_{\rho}^{n m}(T)$ coupling constants at the parameter values $N_{f}=3$, $F=0.100 \mathrm{GeV}$. 


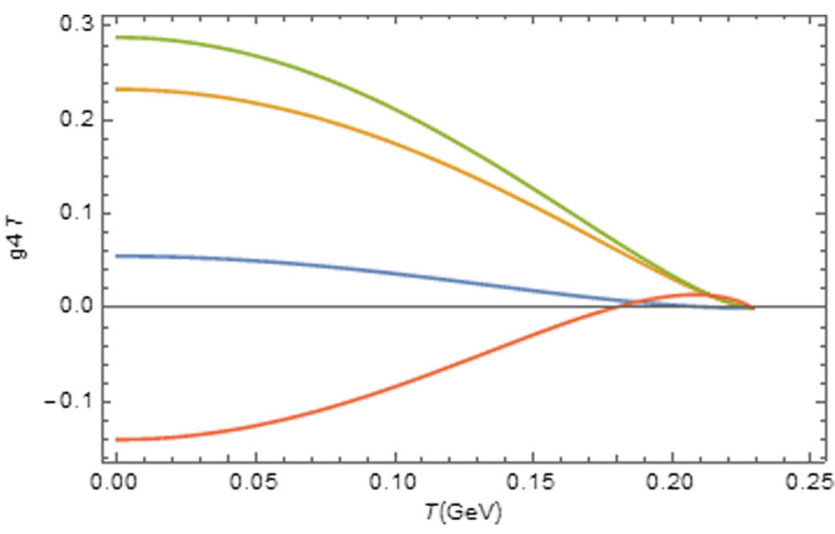

FIG. 3. Temperature dependence of $g_{\rho N N}^{(0) n m}, g_{\rho N N}^{(1) n m}(T), g_{\rho N N}^{(\text {s.w. })}(T)$, and $f_{\rho}^{n m}(T)$ coupling constants at the parameter values $N_{f}=4$, $F=0.130 \mathrm{GeV}$.

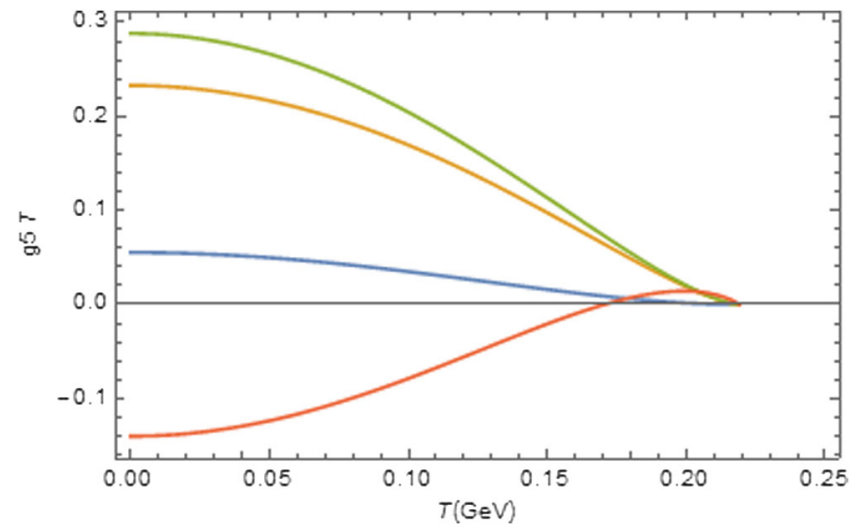

FIG. 4. The $g_{\rho N N}^{(0) n m}, g_{\rho N N}^{(1) n m}(T), g_{\rho N N}^{(\text {s.w. })}(T)$, and $f_{\rho}^{n m}(T)$ coupling constants at the parameter values $N_{f}=5, F=0.140 \mathrm{GeV}$.

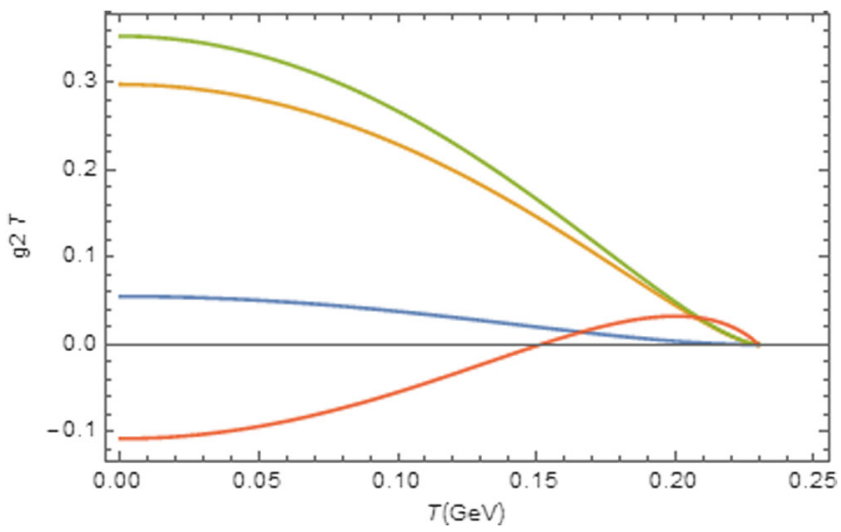

FIG. 5. The $g_{\rho N N}^{(0) n m}, g_{\rho N N}^{(1) n m}(T), g_{\rho N N}^{(\text {s.w. })}(T)$, and $f_{\rho}^{n m}(T)$ coupling constants for the first excited nucleons $N(1440)$ at the parameter values $N_{f}=2, F=0.87 \mathrm{GeV}$.

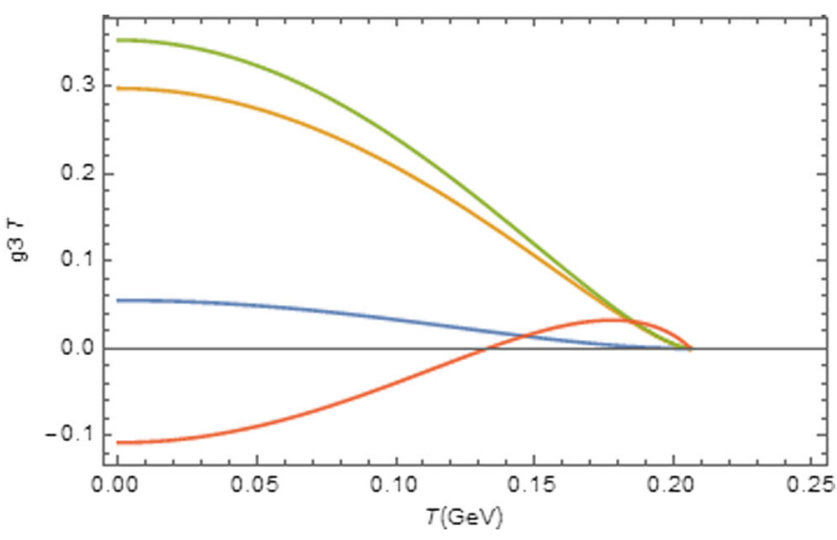

FIG. 6. The $g_{\rho N N}^{(0) n m}, g_{\rho N N}^{(1) n m}(T), g_{\rho N N}^{(\text {s.w. })}(T)$, and $f_{\rho}^{n m}(T)$ coupling constants for the first excited nucleons $N(1440)$ at the parameter values $N_{f}=3, F=0.100 \mathrm{GeV}$.

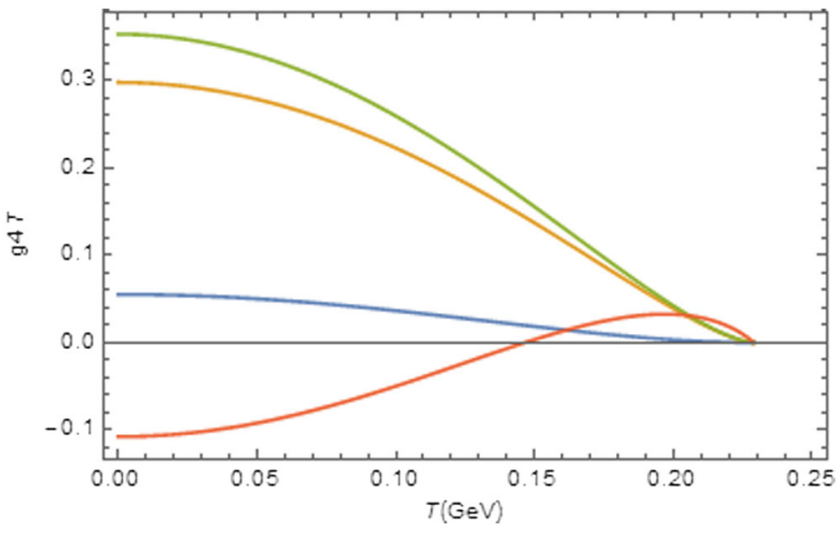

FIG. 7. The $g_{\rho N N}^{(0) n m}, g_{\rho N N}^{(1) n m}(T), g_{\rho N N}^{(\text {s.w. })}(T)$, and $f_{\rho}^{n m}(T)$ coupling constants for the first excited nucleons $N(1440)$ at the parameter values $N_{f}=4, F=0.130 \mathrm{GeV}$.

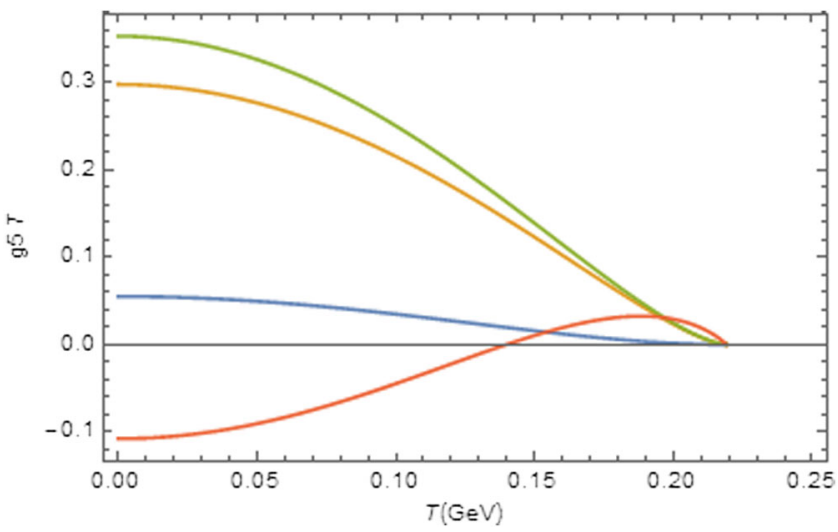

FIG. 8. The $g_{\rho N N}^{(0) n m}, g_{\rho N N}^{(1) n m}(T), g_{\rho N N}^{(\text {s.w. })}(T)$, and $f_{\rho}^{n m}(T)$ coupling constants for the first excited nucleons $N(1440)$ at the parameter values $N_{f}=5, F=0.140 \mathrm{GeV}$. 


\section{DISCUSSION}

In this paper we have studied the temperature dependence of the strong coupling constant of the $\rho$ meson with the nucleons within the soft-wall model of AdS/QCD. We have plotted this dependence for each term in the coupling constant and have observed that all terms become zero at the same point near the Hawking temperature. (This point shifts slightly from case to case, which we think is related to the calculation accuracy.) The result here is reasonable from a physical interpretation point of view. Since the confinementdeconfinement phase transition occurs at the Hawking temperature and there are no hadrons after this temperature, we have obtained a zero value for the coupling constant between the hadrons below this temperature. If we move from the inverse direction of the temperature axis, from higher temperatures to the Hawking temperature, we observe that the $g_{\rho N N}$ coupling constant at the point near the Hawking temperature becomes nonzero not sharply but smoothly. This may be interpreted as follows: strong interactions between the $\rho$ mesons and nucleons emerge not simultaneously with the hadronization, but instead just after the start of cooling of the formed hadron medium. This interpretation may be of use for the understanding processes at the early stages of the formation of the Universe. To have a complete physical picture of the finite temperature interactions between the hadrons, similar investigations are in process for the pion and axial vector-meson interactions with the nucleons in the framework of this formalism.
[1] J. Maldacena, Int. J. Theor. Phys. 38, 1113 (1999); Adv. Theor. Math. Phys. 2, 231 (1998).

[2] E. Witten, Adv. Theor. Math. Phys. 2, 253 (1998).

[3] S. S. Gubser, I. R. Klebanov, and A. M. Polyakov, Phys. Lett. B 428, 105 (1998).

[4] H. Nastase, arXiv:0712.0689.

[5] A. Karch, E. Katz, D. T. Son, and M. A. Stephanov, Phys. Rev. D 74, 015005 (2006).

[6] H. R. Grigoryan and A. V. Radyushkin, Phys. Rev. D 76, 115007 (2007).

[7] Z. Abidin and C. Carlson, Phys. Rev. D 79, 115003 (2009).

[8] T. Gutsche, V. E. Lyubovitskij, I. Schmidt, and A. Vega, Phys. Rev. D 85, 076003 (2012).

[9] P. Colangelo, F. Giannuzzi, and S. Nicotri, J. High Energy Phys. 05 (2012) 076.

[10] P. Colangelo, F. Giannuzzi, S. Nicotri, and F. Zuo, Phys. Rev. D 88, 115011 (2013).

[11] L. A. H. Mamani, A. S. Miranda, H. Boschi-Filho, and N. R. F. Braga, J. High Energy Phys. 03 (2014) 058.

[12] N. R. F. Braga, L. F. Ferreira, and A. Vega, Phys. Lett. B 774, 476 (2017).

[13] S. P. Bartz and T. Jacobson, Phys. Rev. D 94, 075022 (2016).

[14] A. Vega and A. Ibanez, Eur. Phys. J. A 53, 217 (2017).

[15] N. Huseynova and Sh. Mamedov, Int. J. Theor. Phys. 54, 3799 (2015).

[16] N. Huseynova and Sh. Mamedov, Int. J. Mod. Phys. A 34, 1950240 (2019).

[17] S. S. Afonin and I. V. Pusenkov, Eur. Phys. J. C 76, 342 (2016).
[18] J. Chen, S. He, M. Huang, and D. Li, J. High Energy Phys. 01 (2019) 165.

[19] H. J. Kwee and R. Lebed, J. High Energy Phys. 01 (2008) 027.

[20] C. P. Herzog, Phys. Rev. Lett. 98, 091601 (2007).

[21] N. R. F. Braga and L. F. Ferreira, Phys. Lett. B 783, 186 (2018).

[22] N. R. F. Braga, L. F. Ferreira, and R. Da Rocha, Phys. Lett. B 787, 16 (2018).

[23] A. Vega and M. A. M. Contreras, Nucl. Phys. B942, 410 (2019).

[24] X. Cao, S. Qiu, H. Liua, and D. Li, arXiv:2102.10946.

[25] T. Gutsche, V. E. Lyubovitskij, I. Schmidt, and A. Y. Trifonov, Phys. Rev. D 99, 054030 (2019).

[26] T. Gutsche, V. E. Lyubovitskij, I. Schmidt, and A. Y. Trifonov, Phys. Rev. D 99, 114023 (2019).

[27] T. Gutsche, V. E. Lyubovitskij, and I. Schmidt, Nucl. Phys. B952, 114934 (2020).

[28] R. Zöllner and B. Kampfer, Eur. Phys. J. Special Topics 229, 3585 (2020).

[29] X. Cao, S. Qiu, H. Liu, and D. Li, arXiv:2102.10946.

[30] A. Cherman, T. D. Cohen, and E. S. Werbos, Phys. Rev. C 79, 045203 (2009).

[31] N. Maru and M. Tachibana, Eur. Phys. J. C 63, 123 (2009).

[32] H. C. Ahn, D. K. Hong, C. Park, and S. Siwach, Phys. Rev. D 80, 054001 (2009).

[33] K. Chelabi, Z. Fang, M. Huang, D. Li, and Y.-L. Wu, Phys. Rev. D 93, 101901 (2016).

[34] V. G. Stoks and T. A. Rijken, Nucl. Phys. A613, 311 (1997). 